\title{
Low-Dose Strain Mapping by Dark-Field Inline Electron Holography
}

\author{
V.B. Özdöl*, C.T. Koch* and P.A. van Aken* \\ * Stuttgart Center for Electron Microscopy, Max Planck Institute for Metals Research, \\ Heisenbergstr. 3, Stuttgart, Germany, D-70569
}

Be it process-induced strain in the latest generation MOSFETs or biaxial strain in high efficiency optoelectronic structures, optimization of device performance requires quantitative methods for strain analysis. In the last years, dark-field holography techniques have been introduced to overcome the limitations for high-resolution transmission electron microscopy (HRTEM) based methods [1,2], such as limited field of view or requirements for high quality specimens. Unlike dark-field off-axis electron holography [3], dark-field inline holography (DIH) [4] relies on the reconstruction of the geometric phase from a focal series of dark-field images of two non-collinear reflections using, for example, the recently developed fullresolution wave reconstruction (FRWR) software [5]. In contrast to its off-axis counterpart DIH also does not require a Lorentz lens but can be done using the regular objective lens and its aperture.

Apart from its simpler experimental setup and better signal to noise properties, DIH also allows the investigation of electron beam sensitive structures, since, mainly because of the roughly 20 times lower magnification, much lower electron doses are required for image formation than for HRTEM [6]. Fig 1a) displays the subareas of (0002) dark-field Fresnel images of InGaN multi QWs from the active region of a light emitting diode (LED). The reconstructed geometric phase image, and the In distribution map derived from that are shown in Fig 1b) and 1c), respectively. Unlike in HRTEM investigations, where electron beam induced modifications are hardly avoidable, DIH analysis revealed that the InGaN QWs exhibit random alloy nature without any evidence of nanometer scale gross indium clustering in the whole active region.

The present contribution will cover the advances in the data acquisition as well as the basics of the reconstruction algorithm. In addition to the application to real materials problems, recent achievements towards 3D strain mapping will also be demonstrated.

\section{References}

[1] K. Du, F. Phillipp, J. Microsc. 221 (2006) 63.

[2] M. J. Hÿtch, E. Snoeck, and R. Kilaas, Ultramicrosc. 74 (1998) 131.

[3] M. Hÿtch, F. Houdellier, F. Hüe, and E. Snoeck, Nature 453 (2008) 1086109.

[4] C. T. Koch, V. B. Özdöl, and P. A. van Aken, Appl. Phys. Lett. 96 (2010) 091901.

[5] C. T. Koch, Ultramicrosc. 108 (2008) 141109.

[6] V. B. Özdöl, C.T. Koch, and P.A. van Aken, J. Appl. Phys. 108 (2010) 056103.

[7] We acknowledge financial support from the European Union under the FP6 Integrated Infrastructure Initiative, Reference 026019 (ESTEEM). 
a)

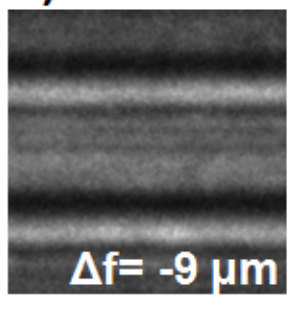

b)

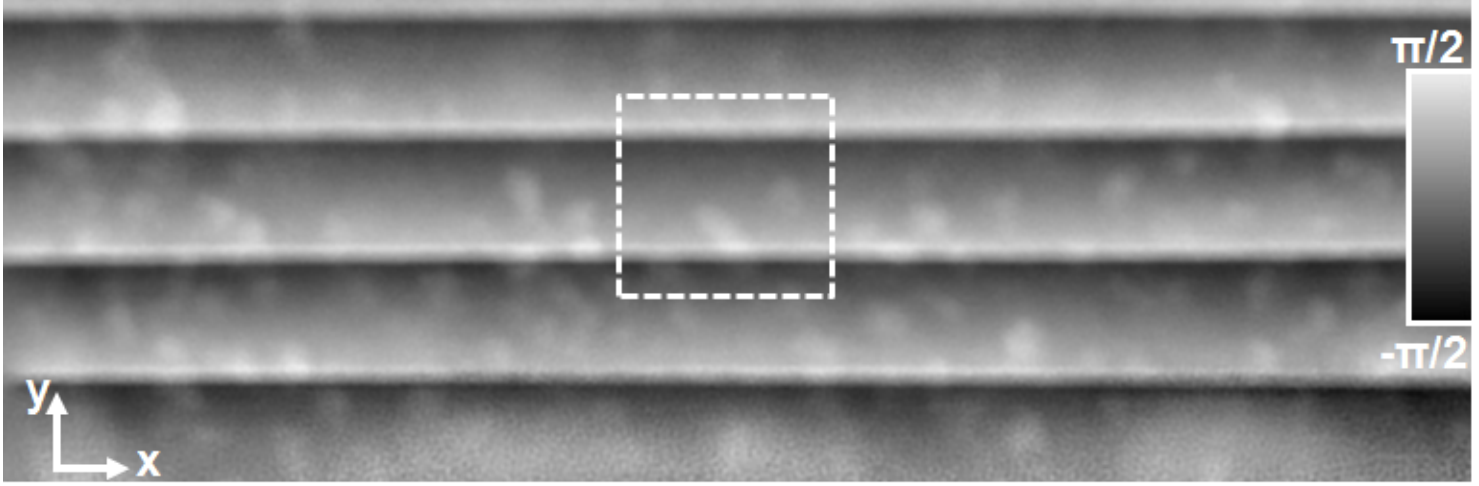

c)
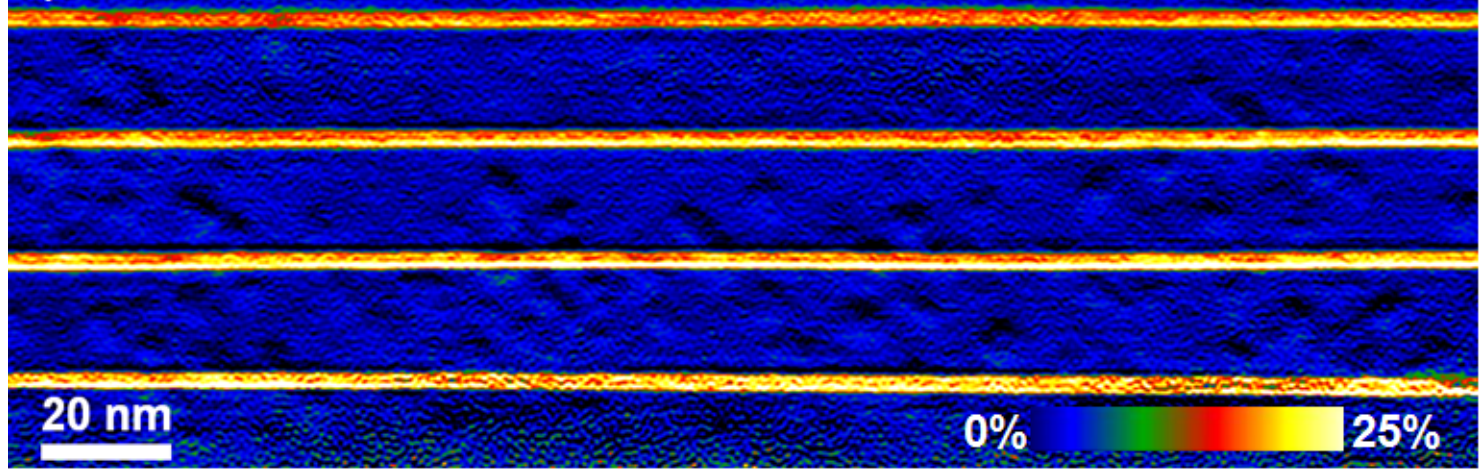

FIG. 1. (a) Subareas of the (0002) dark-field Fresnel images of two InGaN QWs acquired at different defocus values. (b) Geometric phase reconstructed from a focal series of 11 dark-field images covering the whole active region of the LED. The size of the dashed square corresponds to the size of the subareas of the dark field images. (c) Indium composition map derived from the geometric phase image reveals homogenous distribution without any evidence of nanometer scale gross indium clustering. 